\title{
CRITERES PHYSIOLOGIQUES DISCRIMINANTS AU JEUNE AGE POUR LA SELECTION DE GENOTYPES DE PALMIER A HUILE (Elaeis guineensis JACQ.) TOLERANTS A LA SECHERESSE
}

\author{
O. NDIAYE ${ }^{1}$, O. DIOUF ${ }^{2}$, B. ADON ${ }^{1}$ et S. BRACONNIER ${ }^{2}$
}

\author{
${ }^{1}$ Station de recherche palmier à huile (CNRA) 13 BP 989 Abidjan 13 COTE D'IVOIRE \\ E-mail : n_oumar@hotmail.com
}

\begin{abstract}
${ }^{2}$ Centre d'étude régional pour l'amélioration de l'adaptation à la sécheresse (CERAAS) / Centre de coopération internationale en recherche agronomique pour le développement (CIRAD) BP 3320, Thiès Escale, THIES - SENEGAL
\end{abstract}

\section{RESUME}

L'alimentation hydrique est le principal facteur limitant la productivité du palmier à huile (Elaeis guineensis Jacq.). Les réponses physiologiques des plantules, soumises à un déficit hydrique, ont été évaluées sous serre, dans des pots durant 4 mois. En vue d'identifier des critères discriminants au jeune âge pour une utilisation en sélection variétale, du matériel sensible et un autre tolérant à la sécheresse ont été soumis à un cycle de dessèchement - réhydratation. Les paramètres tels que : le statut hydrique (taux de transpiration relative, potentiel hydrique foliaire), les échanges gazeux (photosynthèse, conductance stomatique) et l'activité photochimique (fluorescence chlorophyllienne) ont été étudiés. Le régime hydrique a fortement affecté l'ensemble des paramètres étudiés. Le taux de satisfaction des besoins en eau a varié de 90 à $4 \%$, et le potentiel hydrique a atteint une valeur de $-3 \mathrm{MPa}$ trois semaines après suspension de l'arrosage. Pendant la phase de réhydratation, les plantules ont présenté une capacité de reprise satisfaisante. Malgré une bonne corrélation, les deux paramètres d'échanges gazeux ne peuvent être proposés isolément comme critères de sélection dans les conditions de l'expérience. Par contre, la mesure de la fluorescence chlorophyllienne a permis de distinguer le matériel tolérant du sensible au bout d'une semaine. Ces resultats ouvrent une perspective dans la sélection de génotypes de palmier à huile tolérants à la sécheresse au jeune âge.

Mots-clés : Elaeis guineensis, palmier à huile, déficit hydrique, photosynthèse, fluorescence chlorophyllienne, Côte d'Ivoire.

\author{
ABSTRACT \\ ASSESSMENT OF PHYSIOLOGICAL RESPONSES OF YOUNG OIL PALM (Elaeis guineensis JACQ.) GENOTYPES FOR \\ EARLYSCREENING TODROUGHT TOLERANCE
}

Water deficit is the main limiting factor in oil palm production (Elaeis guineensis Jacq.) in West Africa. Physiological responses to drought plantlets were accessed under green house conditions for 4 months. $A$ drought tolerant species and a sensitive one were subject to one wetting and drying cycle. Parameters such as water status (transpiration rate, waterpotential), leafgas exchanges (photosynthesis, stomatalconductance) and photochemical activity (chlorophyll fluorescence) were monitored. Results show that all parameters were affected by watering. Transpiration rate dropped from 90 to $4 \%$, with a leaf potential close to $-3 \mathrm{MPa}$ three weeks after watering was interrupted. Despite a good correlation, the two leaf gas exchange parameters alone were not relevant criteria for the early screening of oil palm material. However, chlorophyll fluorescence data allowed early screening of both genotypes within one week after withdrawal of watering. The latter parameter can offer effective tool to oil palm breeders for screening young oil palm genotypes to drought tolerance.

Key-words : Elaeis guineensis, oil palm, water deficit, photosynthesis, chlorophyll fluorescence, Côte d'Ivoire. 


\section{INTRODUCTION}

Bien que l'origine du palmier à huile soit l'Afrique de l'ouest, les conditions climatiques de la zone ne sont pas toujours favorables à une production optimale. Parmi celles-ci, l'eau est le facteur du milieu qui a la plus grande importance pour l'expression du potentiel de production de cette plante. Plusieurs auteurs ont étudié l'influence du déficit hydrique, sur les composantes du rendement du palmier à huile (Adam, 1910 ; Devuyst, 1948 ; Surre, 1968 ; Corley, 1983, Dufour et al., 1988 ; Caliman, 1992 ; Nguettia et al., 1995 ; Quencez, 1996 ; Nouy et al., 1999). Les besoins en eau de la culture sont très élevés et peuvent atteindre 1800 à $2400 \mathrm{~mm}$ d'eau par an, bien repartis dans l'année. Mais ces besoins sont rarement couverts par la pluviométrie et la sécheresse a un effet négatif sur la croissance de la plante, le sex-ratio, le taux d'avortement des inflorescences femelles et la production (Caliman, 1992). En cas de déficit hydrique sévère $(>350 \mathrm{~mm})$ on note une baisse importante de la production, de 24 à $5,5 \mathrm{t}^{\text {ha }}{ }^{-1}$ $a^{-1}$ et le nombre d'inflorescences mâles produites augmente considérablement (Nguettia et al., 1995). Dans les cas extrêmes, l'arbre meurt. Malgré cette relative sensibilité du palmier à huile au déficit hydrique, il existe des palmiers au champ qui associent un niveau de productivité, à un niveau de tolérance satisfaisants vis-à-vis de la sécheresse.

Cependant, comme la plupart des plantes pérennes, l'étude des facteurs de résistance à la sécheresse reste difficile au champ. En effet, si l'on veut des résultats de production, il faut attendre que les plants soient adultes, et âgés de 12-14 ans. De plus, chez le palmier à huile, la durée entre l'initiation florale et la récolte du régime est d'environ 26 mois (Nguettia et al., 1995). II s'avère alors très difficile de corréler les rendements à des aléas climatiques qui peuvent remonter parfois à plus de 2 ans, sachant que d'autres facteurs ont également pu intervenir par la suite. Tout cela rend l'étude de l'influence du déficit hydrique sur la production du palmier à huile, relativement compliquée. L'idée d'identifier des caractères au jeune âge, capables de différencier des génotypes plus tolérants que d'autres à la sécheresse, est très intéressante, puisqu'elle permettrait d'accélérer les cycles de sélection du fait d'un criblage précoce. L'objectif de cette étude est donc de caractériser, au stade pépinière, les réponses physiologiques du palmier à huile à travers l'état hydrique, les échanges gazeux foliaires et la fluorescence chlorophyllienne. Elle vise à proposer des critères précoces utilisables en sélection pour le choix de génotypes tolérants à la sécheresse.

\section{MATERIEL ET METHODES}

\section{SITE EXPERIMENTAL}

L'essai a été réalisé sous serre au CERAAS (Centre d'Etude Régional pour l'Amélioration de l'Adaptation à la Sécheresse) situé à $4 \mathrm{Km}$ de Thiès $\left(14^{\circ} 42^{\prime} \mathrm{N}, 16^{\circ} 28^{\prime} \mathrm{W}\right)$ au Sénégal. La température moyenne et l'humidité relative ont été enregistrées quotidiennement, au pas de temps horaire, grâce à un capteur d'humidité. La photopériode a été proche de $12 \mathrm{~h}$ (entre $7 \mathrm{~h}$ et $19 \mathrm{~h}$ ). La Figure 1 montre une température moyenne horaire entre 27 et $30^{\circ} \mathrm{C}$, alors que l'humidité fluctue entre $90 \%$, en fin de nuit, et $60 \%$ à la mi-journée. Ces conditions sont favorables aux palmiers à huile.



Figure 1 : Evolution horaire de l'humidité relative et de la température dans la serre. (Moyenne mobile horaire) entre $7 \mathrm{~h}$ et $19 \mathrm{~h}$.

Changes in relative humidity and temperature within the green house used for the experiment. (Average hours) between 7 and $19 \mathrm{~h}$. 


\section{MATERIEL VEGETAL}

Deux génotypes de palmier à huile issus des croisements entre les géniteurs LM2T et DA10D d'une part, et LM2T et LM404D d'autre part, ont été utilisés dans cette étude. Le géniteur DA10D se caractérise au champ par une tolérance à la sécheresse, à la différence de LM404D, plutôt sensible. Les deux génotypes testés ont été obtenus par fécondation artificielle sur la station de recherche de La Mé en Côte d'Ivoire.

Les graines ont été repiquées dans des pots de culture en gaines de polyéthylène noires de 25 $\mathrm{cm}$ de haut sur $20 \mathrm{~cm}$ de diamètre et $5 / 100^{\circ}$ d'épaisseur, perforées de 20 trous à la base. Chaque pot a été rempli de $5 \mathrm{~kg}$ de terre préalablement tamisée $(\varnothing=2 \mathrm{~mm})$. Au bout de 90 jours, 40 plantules par génotypes ont été retenues en fonction des critères de sélection en pépinière (Jacquemard, 1995) et de I'homogénéité de leur état végétatif moyen.

\section{TRAITEMENT HYDRIQUE}

L'expérimentation a porté sur deux régimes hydriques :

- un témoin maintenu à la capacité au champ durant tout l'essai. Les doses journalières d'irrigation ont été obtenues par des pesées quotidiennes pour compenser exactement par différence de poids, les pertes d'eau de la veille du pot contenant la plantule. L'apport d'eau a été fait manuellementà l'aide d'un récipient gradué.
- un traitement stressé, dont les plantules ont été soumises à un déficit hydrique obtenu par suspension d'arrosage durant 21 jours. Les plantules ont ensuite été réhydratées pendant $4 \mathrm{j}$ en ramenant le sol des pots à la capacité au champ. Le cycle desséchement-réhydratation a donc duré $25 \mathrm{j}$.

\section{DISPOSITIF EXPERIMENTAL}

Le dispositif expérimental est un bloc de Fisher permettant l'étude de deux facteurs à deux niveaux : le matériel végétal (génotypes LM2T $x$ DA10D et LM2T $x$ LM404D), et le traitement hydrique (témoin et suspension d'arrosage pendant 21 jours puis réhydratation pendant 4 jours). Le dispositif comportait 5 répétitions, et chaque unité expérimentale était constituée de deux plantules.

Les analyses statistiques ont été faites avec le logiciel SAS (SAS Institut, 1989). Les différences entre traitements ont été considérées comme statistiquement significatives au seuil de $5 \%$.

\section{OBSERVATIONS PHYSIOLOGIQUES}

L'ensemble des observations physiologiques a porté sur des plantules âgées de 4 mois. A ce stade, chaque plantule portait au moins 3 à 4 feuilles lancéolées bien ouvertes (Figure 2).

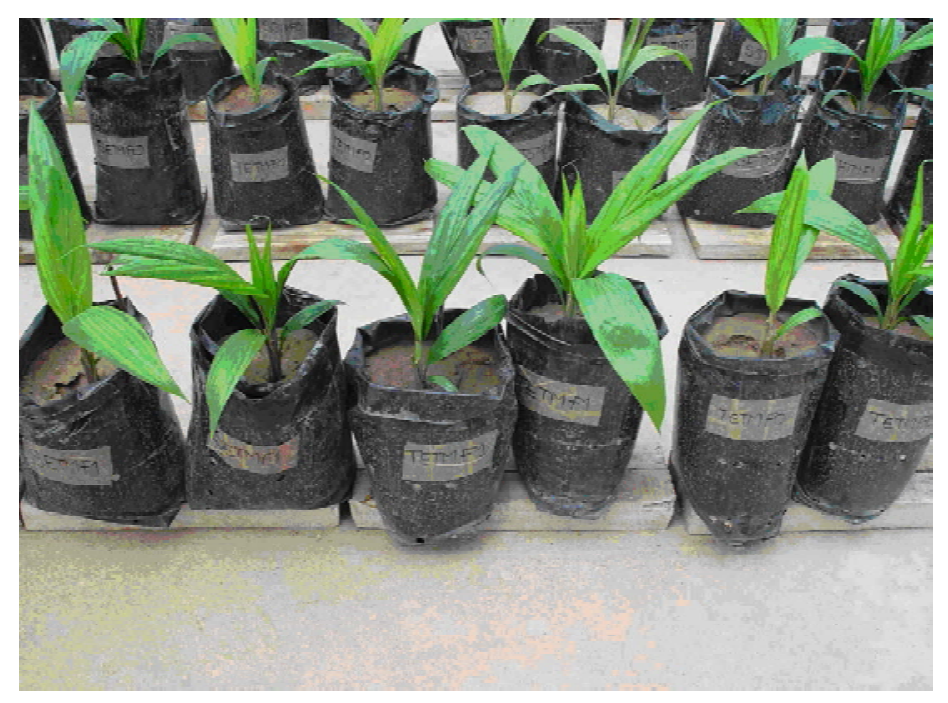

Figure 2 : Vue générale de plantules de palmier à huile âgées de 4 mois. $A$ view of 4-month-old oil plam plants. 


\section{Caractérisation de l'état hydrique des plantules}

\section{Satisfaction des besoins en eau}

Le taux de transpiration relative (TTR) a été retenu comme critère d'appréciation de l'état de déshydratation des plantules (Wormer et Ochs, 1959 ; Adjahoussou, 1977 ; Dufrene, 1989 ; Dufrene et al., 1992). Ce paramètre a permis également de suivre la cinétique d'installation du stress hydrique et la réaction physiologique des palmiers à ce phénomène.

Le taux de transpiration relative renseigne sur le niveau de satisfaction des besoins en eau, et correspond au rapport de l'évapotranspiration réelle (ETR) sur l'évapotranspiration maximale (ETM) à la même date, exprimé en pourcentage.

$\operatorname{TTR}(\%)=(E T R / E T M) \times 100$

L'ETR a été déterminée par la méthode de pesées journalières (différence de poids entre deux pesées successives de chaque pot). Par simplification, l'ETM a été ramenée à la valeur maximale de l'évapotranspiration relevée à chaque date de mesure sur l'ensemble des 40 pots de l'essai. Toutes les mesures ont été effectuées entre 8 et $9 \mathrm{~h}$ du matin, afin de les coupler avec les mesures des autres paramètres physiologiques. Ainsi, à chaque date de mesure, l'état de déshydratation moyen des plantules a été déterminé.

\section{Potentiel hydrique foliaire}

Le potentiel hydrique foliaire ( $\Psi$ h) renseigne sur l'état de liaison de l'eau dans la cellule et l'énergie qu'il faudrait pour l'en extraire et la porter à l'état libre (Cruizat, 1974 ; Diouf, 1993). II a été mesuré avec une chambre à pression SCHOLANDER de type PMS modèle 650 (PMS Instrument Co, Corvalis Oregon USA). Cet appareil est couramment utilisé sur le palmier à huile (Adjahoussou, 1977 ; Dufrène, 1989 ; Villalobos et al., 1992) et sur le cocotier (Konan, 1993 ; Repellin et al., 1997). Sur chaque plantule, la feuille $n^{\circ} 4$ a été excisée et placée immédiatement dans la chambre de mesure pour faire la lecture. Les observations ont été effectuées au $14^{\mathrm{e}}$ et au $21^{\mathrm{e}} \mathrm{j}$ de stress, puis $4 \mathrm{j}$ après la réhydratation des plantules.

\section{Mesure des échanges gazeux foliaires}

Les échanges gazeux foliaires ont été mesurés en circuit ouvert grâce à un analyseur portable de type Licor modèle LI-6400 (Licor Inc., Lincoln, Nebraska,USA). Les conditions de mesure dans la chambre ont été les suivantes : rayonnement photosynthétiquement actif incident (PAR) égal à $1200 \mu \mathrm{mol} \mathrm{m}{ }^{-2} \mathrm{~s}^{-1}$, température à $30^{\circ} \mathrm{C}$ et concentration en $\mathrm{CO}_{2}$ à $350 \mathrm{vpm}$. Toutes les mesures ont été effectuées, autant que possible, entre $10 \mathrm{~h}$ et $12 \mathrm{~h}$ le matin et ont porté sur la feuille $n^{\circ} 4$, considérée chez le palmier à huile comme la première feuille physiologiquement stable à cet âge (Dufrène et Saugier, 1993). Enfin, les mesures ont été effectuées à une humidité relative toujours supérieure à $65 \%$ pour éviter un effet dépressif possible du déficit de pression de vapeur de l'air sur la photosynthèse (Dufrène, 1989).

\section{Mesure de la fluorescence chlorophyllienne}

Les mesures de fluorescence chlorophyllienne ont été réalisées in vivo sur les feuilles au moyen d'un fluorimètre de type PEA (Plant Efficiency Analyser, Hansatech Inst. Ltd, King's Lynn, England). La technique de mesure et la méthode de calcul des paramètres de fluorescence chlorophyllienne ont été décrites par Strasser et Strasser (1995) et Krüger et al. (1997). L'activité photochimique des chloroplastes et les changements de fluorescence chlorophyllienne qui lui sont associés, ont été induits par l'application d'un flash lumineux rouge $(650 \mathrm{~nm})$ d'intensité $3000 \mu \mathrm{mol} \mathrm{m} \mathrm{m}^{-2} \mathrm{~s}^{-1}$ émis par 6 diodes. Les feuilles ont été préalablement adaptées à l'obscurité à l'aide d'un clip (pince) pendant environ 30 minutes. La mise à l'obscurité est nécessaire pour la réouverture des centres réactionnels des chloroplastes, de sorte que l'induction de fluorescence puisse refléter leur fermeture par la lumière. Les paramètres de fluorescence chlorophyllienne retenus sont:

- le rendement quantique maximal du photosystème II (PS II) noté $\varphi \mathrm{PO}$. II mesure la probabilité pour qu'un photon absorbé soit piégé par un centre réactionnel du PS II.

- Le rendement de transfert d'électrons le long de la chaîne de réaction photosynthétique, noté $\Psi 0$. Ce paramètre désigne la probabilité pour qu'un photon absorbé par le centre réactionnel, déplace un électron au-delà de la Quinone $A$ $\left(Q_{A}\right)$.

- L'indice de vitalité du chloroplaste noté SFI (Structure Function Index). C'est un paramètre intégré qui fournit des informations à la fois sur 
le fonctionnement et sur l'intégrité des chloroplastes au niveau de la surface foliaire qui reçoit le flash lumineux.

Ces trois paramètres ont été retenus pour leur assez bonne corrélation avec l'assimilation de $\mathrm{CO}_{2}$ et le stress hydrique (Cerovic et al., 1996 ; He et Edwards, 1996 ; Flexas et al., 2000).

\section{RESULTATS}

\section{SATISFACTION DES BESOINS EN EAU}

L'évolution du taux de transpiration relative est présentée à la figure 3. L'installation du stress a été rapide et brutale jusqu'au $10^{\mathrm{e}} \mathrm{j}$, puis plus régulière durant les 10 derniers jours. L'effet du régime hydrique sur la satisfaction des besoins en eau a été significatif dès le $2^{\mathrm{e}} \mathrm{j}$ de stress $(\alpha<0,05)$ et la différence avec le témoin s'est accrue jusqu'à la fin de la $3^{e}$ semaine $(\alpha<0,0001)$. L'analyse statistique des résultats n'a pas permis de mettre en évidence un effet significatif du facteur matériel végétal sur ce paramètre.

La réhydratation de $4 \mathrm{j}$ a permis aux plantules de récupérer plus de la moitié (52\%) de leur teneur en eau optimale, mais, là encore, sans distinction significative entre les deux génotypes.

\section{POTENTIEL HYDRIQUE FOLIAIRE $(\Psi H)$}

L'évolution du potentiel hydrique au cours de l'expérimentation est exposée à la figure 4. La suspension de l'arrosage a entraîné une baisse significative des valeurs du $\Psi \mathrm{h}$. Les valeurs minimales ont été atteintes 3 semaines après l'arrêt de l'irrigation, et sont restées inférieures à -3 $\mathrm{MPa}$, témoignant d'un état de stress sévère pour des plantules de palmier à huile âgées de 4 mois. Là encore, le matériel végétal n'a pas d'effet significatif sur ce paramètre.

Après $4 \mathrm{j}$ de réhydratation, le potentiel hydrique des plantules stressées n'a pas été significativement différent de celui des témoins irrigués.

\section{ECHANGES GAZEUX CHLOROPHYLLIENS}

Les observations faites au cours de l'expérimentation sont consignées dans le tableau 1. Pendant toute la phase de dessèchement du sol, aucune différence significative n'a été observée entre le matériel tolérant et le matériel sensible. Par contre, le régime hydrique a eu un effet dépressif hautement significatif $(\alpha<0,0001)$ sur l'assimilation de $\mathrm{CO}_{2}$ dès le $2^{\mathrm{e}} \mathrm{j}$ de stress. Puis, l'écart entre traitements irrigués et stressés s'est accentué jusqu'au $14^{\mathrm{e}} \mathrm{j}$. A cette date, la photosynthèse moyenne des traitements stressés ne représentait plus que $6 \%$ des témoins irrigués, avec une valeur moyenne de $0,75 \mu \mathrm{mol} \mathrm{m}^{-2} \mathrm{~s}^{-1}$. En $4 \mathrm{j}$ de réhydratation, la photosynthèse est passée de $0,75 \mu \mathrm{mol} \mathrm{m} \mathrm{m}^{-2} \mathrm{~s}^{-1}$ (au $14^{\mathrm{e}} \mathrm{j}$ de stress) à $2,34 \mu \mathrm{mol} \mathrm{m} \mathrm{m}^{-2} \mathrm{~s}^{-1}$, mais est restée très inférieure à la valeur moyenne observée chez les plants témoins. Ceci prouve que la récupération du palmier à huile au stade plantule est relativement lente après un stress sévère.

L'évolution de la conductance stomatique au cours du stress a été comparable à celle de la photosynthèse. En effet, le régime hydrique a eu un effet hautement significatif $(\alpha<0,0001)$ à partir du $8^{\mathrm{e}} \mathrm{j}$, avec une baisse de près de $60 \%$ des valeurs mesurées chez les plantules stressées, par rapport aux témoins. Au $14^{\mathrm{e}} \mathrm{j}$, la conductance stomatique des plantules stressées ne représentait plus que $5 \%$ de celle des témoins. La période de réhydratation a permis aux valeurs de la conductance stomatique de passer de $0,15 \mathrm{~mol} \mathrm{~m}^{-2} \mathrm{~s}^{-1}$ (au $14^{\mathrm{e}} \mathrm{j}$ de stress) à $0,32 \mathrm{~mol} \mathrm{~m}^{-2} \mathrm{~s}^{-1}$.

L'étude de la relation entre la photosynthèse et la conductance stomatique a permis de mettre en évidence une différence de comportement entre les deux génotypes de palmier à huile (Figure 5). Le matériel tolérant a présenté une meilleure dispersion des points par rapport au matériel sensible à la sécheresse. En effet, chez ces derniers, on a pu distinguer nettement les plantules irriguées ( $\mathrm{Gs}>0,3 \mathrm{~mol} \mathrm{~m}^{-2} \mathrm{~s}^{-1}$ ) des plantules stressées ( $\mathrm{Gs}<0,1 \mathrm{~mol} \mathrm{~m}^{-2} \mathrm{~s}^{-1}$ ). En revanche, pour le matériel tolérant, la gamme a varié des conductances les plus faibles aux plus élevées. Par conséquent, en conditions d'alimentation hydrique non limitantes et pour des niveaux identiques de photosynthèse, LM404D (sensible) a eu des valeurs de conductance stomatique régulièrement plus élevées que DA10D (tolérant). Ce résultat laisse penser que l'efficience d'utilisation de l'eau est moins élevée chez le matériel sensible que chez le tolérant. 


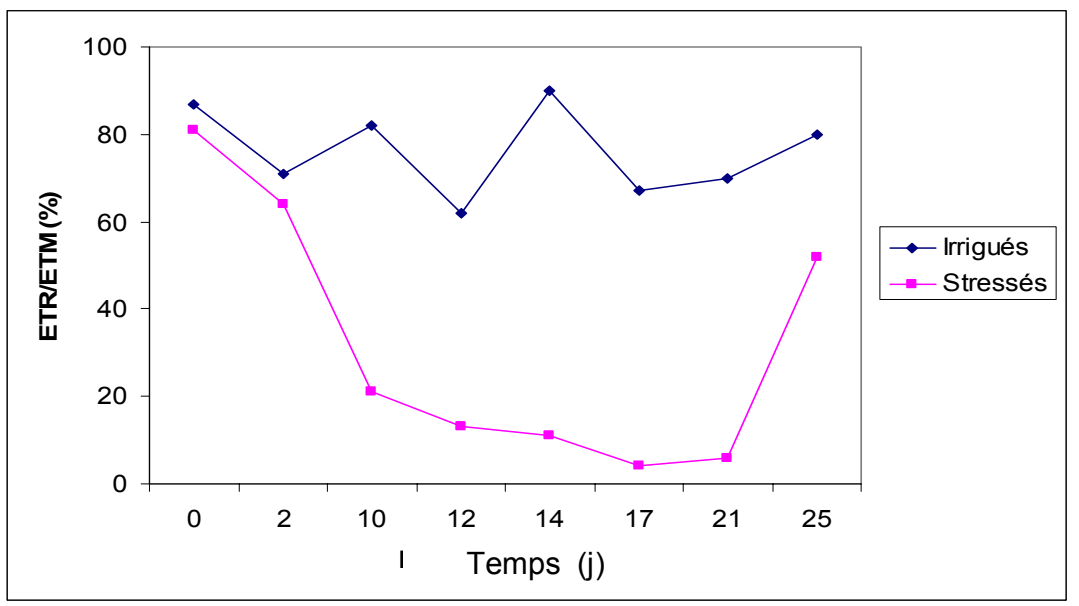

Figure 3 : Evolution de la transpiration relative (ETR/ETM) des plantules de palmier à huile (4 mois) au cours d'un cycle de dessèchement -réhydratation.

Changes in relative transpiration of 4-month-old oil palm plants during a wetting drying cycle.

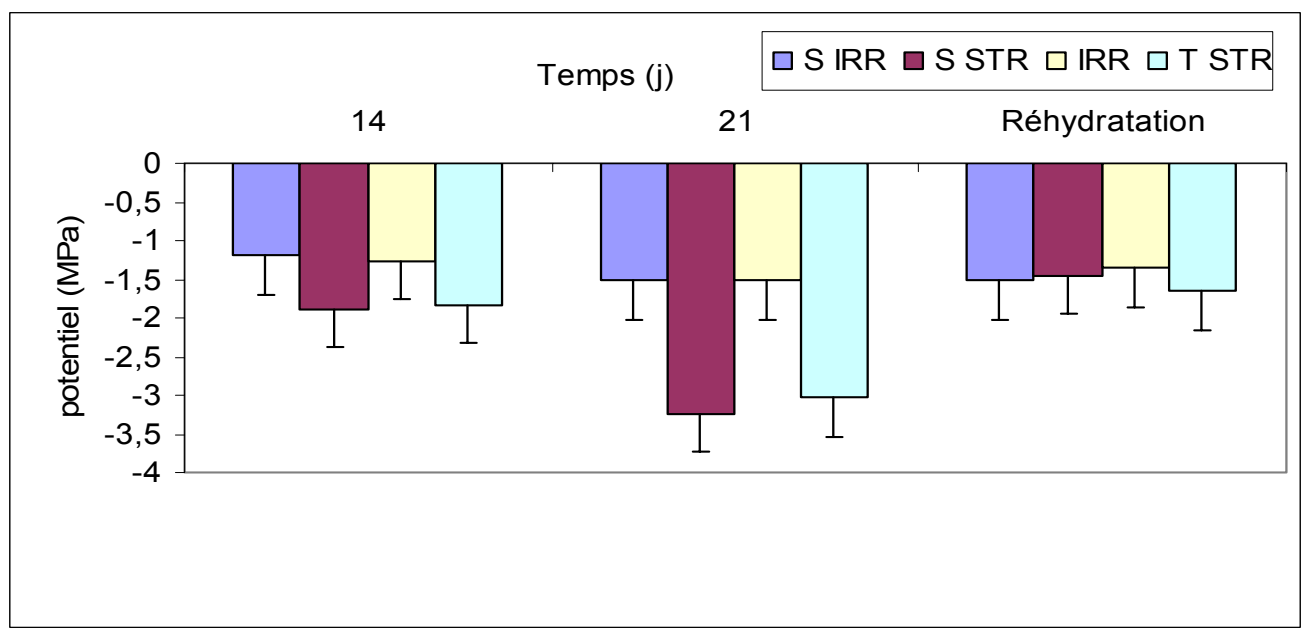

Figure 4 : Potentiel hydrique foliaire $(\Psi \mathrm{h})$ des plantules de palmiers à huile en fonction du temps.

Leaf water potential $(\Psi \mathrm{h})$ of young oil palm plants as function of time.

( $S=$ LM404D x LM2T ; T = DA10D x LM2T ; IRR = plantules irriguées à la capacité au champ ; STR = plantules stressées).

Tableau 1 : Variations de la photosynthèse $\left(\mu \mathrm{mol} \mathrm{m}^{-2} \mathrm{~s}^{-1}\right)$ de plantules de palmier à huile âgées de 4 mois au cours du stress.

Variation in photosynthetisis activity of 4-month-old oil palm plants during water stress.

\begin{tabular}{ccccccc}
\hline \multirow{2}{*}{ Paramètres } & \multicolumn{6}{c}{ Variation de la photosynthèse selon le nombre de jours de stress $\left(\mu \mathrm{mol} \mathrm{m}^{-2} \mathrm{~s}^{-1}\right)$} \\
\cline { 2 - 7 } & 0 & 2 & 4 & 8 & 12 & 14 \\
\hline Matériel végétal & 12,98 & 14,83 & 12,62 & 12,00 & 8,91 & 7,35 \\
DA10D & 13,25 & 13,74 & 11,93 & 11,64 & 8,37 & 6,81 \\
LM404D & -2 & +7 & +5 & +3 & +6 & +7 \\
\hline Ecarts (\%) & 13,23 & 15,03 & 13,01 & 14,95 & 13,57 & 13,40 \\
\hline Régime hydrique & 12,99 & 13,53 & 11,54 & 8,70 & 3,72 & 0,75 \\
Irrigués & -2 & $-10^{*}$ & $-12^{*}$ & $-42^{* * * *}$ & $-73^{* * * *}$ & $-94^{* * * *}$ \\
Stressés & & & & & & \\
\hline Ecarts (\%) & &
\end{tabular}

$*$ seuil $\alpha<0,05 ; * * \star *=$ seuil $\alpha<0,0001$ 


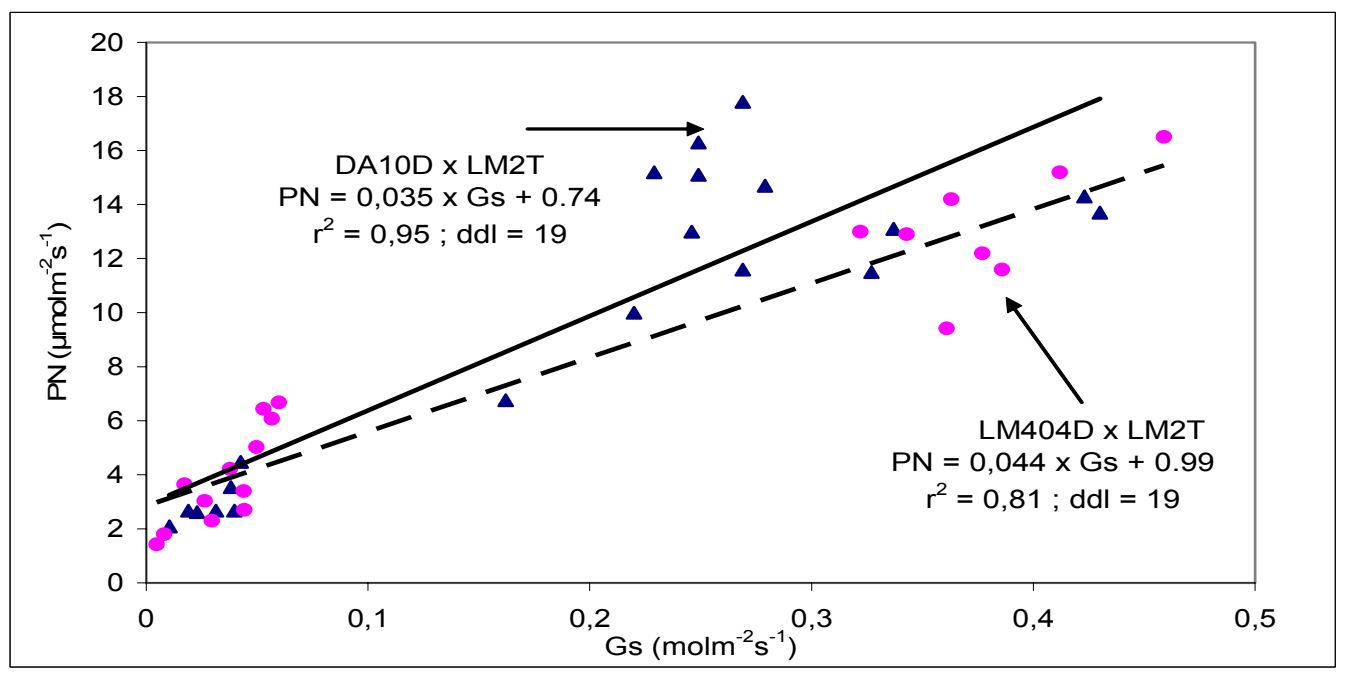

Figure 5 : Relation entre la conductance (Gs) et la photosynthèse (PN) pour deux origines génétiques de palmier à huile âgée de 4 mois.

Relationship between conductance and photosynthesis for two 4-month-old oil palm plants varieties.

\section{ACTIVITE PHOTOCHIMIQUE}

Le rendement quantique maximal ( $\varphi P 0)$ du PSII a été significativement affecté par le régime hydrique $(\alpha<0,0001)$ à partir du $17^{\mathrm{e}} \mathrm{j}$ de stress. Cela s'est traduit par une baisse de 24 à $60 \%$ entre le $17^{\mathrm{e}}$ et le $21^{\mathrm{e}} \mathrm{j}$ entre plantules irriguées et stressées (Figure 6). Le rendement du transfert d'électrons $(\Psi 0)$ nécessaire à l'accomplissement de l'activité photochimique primaire, a été influencé par l'interaction matériel végétal $x$ régime hydrique $x$ date $(\alpha<0,05)$. $A$ ce niveau, l'effet du régime hydrique a été différent dans le temps selon le génotype. En effet chez le matériel sensible, le stress hydrique a affecté le rendement du transfert d'électrons dès le $7^{e} \mathrm{j}$. Pourtant chez le matériel tolérant, cet effet n'a été observé qu'à partir du $17^{\mathrm{e}} \mathrm{j}$ (Tableau 2). L'indice de vitalité $(\mathrm{SFI})$ s'est révélé être un indicateur efficacité de l'intégrité et de la fonctionnalité de l'appareil chloroplastique (Tableau 3). Une triple interaction matériel végétal $\mathrm{x}$ régime hydrique $\mathrm{x}$ date $(\alpha<0,05)$ a été également observée. Comme pour $\Psi 0$, on peut penser que l'effet du régime hydrique pourrait avoir influencé le comportement des plantules dans le temps. En effet, le même comportement dans le temps chez les deux génotypes a été observé, comme pour le paramètre précédent. Pendant la phase de réhydratation, l'effet dépressif du stress hydrique a persisté sur $\varphi \mathrm{PO}$ et $\mathrm{SFI}$ malgré le retour des plantules à l'irrigation complète pendant $4 \mathrm{j}$. L'écart entre traitements stressés et témoins irrigués a été de $-16 \%(\alpha<0,05)$ pour $\varphi P 0$ et $-43 \%$ pour SFI $(\alpha<0,01)$. Toutefois, il s'agit là d'un niveau de récupération acceptable, puisqu'au $21^{\mathrm{e}} \mathrm{j}$ de stress ces deux valeurs ont été respectivement de - $60 \%$ et - $92 \%$ $(\alpha<0,0001)$.

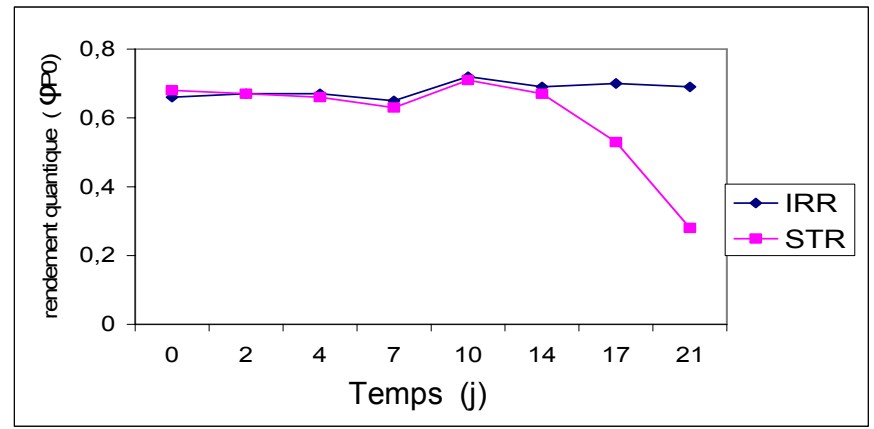

Figure 6 : Rendement quantique maximal $(\varphi P 0)$ du palmier à huile (4 mois) en fonction du régime hydrique.

Maximum quantum yield of a 4-month-old oil palm plants as a function of water regimes. (IRR = plantules irriguées à la capacité au champ ; STR = plantules stressées).

$(I R R=$ irrigated plants at field capacity, STR $=$ water stressed plants $)$. 
Tableau 2 : Rendement du transfert d'électrons $(\Psi 0)$ chez le palmier à huile âgées de 4 mois en fonction du régime hydrique.

Electron transfert yield in a 4-month-old oil palm plants as a function of the water regime.

\begin{tabular}{lcccccccc}
\hline & \multicolumn{7}{c}{ Variation du transfert d'électrons selon le nombre de jours } \\
\cline { 2 - 8 } Paramètres & 0 & 2 & 4 & 7 & 10 & 14 & 17 & 21 \\
\hline LM404D & & & & & & & & \\
Irrigué & 0,37 & 0,35 & 0,36 & 0,36 & 0,46 & 0,40 & 0,39 & 0,43 \\
Stressé & 0,37 & 0,33 & 0,33 & 0,25 & 0,35 & 0,28 & 0,25 & 0,18 \\
\hline Ecart (\%) & 0 & -5 & -8 & $-31^{* * * *}$ & $-24^{*}$ & $-30^{* * * *}$ & $-36^{* * * *}$ & $-58^{* * * *}$ \\
\hline DA10D & & & & & & & & 0,34 \\
Irrigué & 0,43 & 0,41 & 0,40 & 0,38 & 0,39 & 0,36 & 0,37 & 0,36 \\
Stressé & 0,42 & 0,40 & 0,40 & 0,37 & 0,38 & 0,36 & 0,26 & 0,19 \\
\hline Ecart (\%) & -2 & -2 & 0 & -3 & -3 & 0 & $-30^{* * * *}$ & $-44^{* * * *}$ \\
\hline
\end{tabular}

$\left({ }^{\star}=\right.$ seuil $\alpha<0,01 ;{ }^{* * * *}=$ seuil $\left.\alpha<0,0001\right)$

Tableau 3 : Indice de vitalité des plantules de palmier à huile âgées de 4 mois en fonction du régime hydrique.

Vitality index of 4-month old oil palm plants as a function of the water regime.

\begin{tabular}{lcccccccc}
\hline & \multicolumn{7}{c}{ Indice de vitalité en fonction du nombre de jours } \\
\cline { 2 - 9 } Paramètres & 0 & 2 & 4 & 7 & 10 & 14 & 17 & 21 \\
\hline LM404D & & & & & & & & \\
Irrigué & 0,81 & 0,83 & 0,90 & 0,83 & 1,33 & 1,12 & 1,14 & 1,28 \\
Stressé & 0,93 & 1,06 & 0,78 & 0,51 & 0,96 & 1,00 & 0,50 & 0,19 \\
\hline Ecart (\%) & +15 & +28 & -13 & $-39^{*}$ & $-28^{*}$ & $-11^{* *}$ & $-56^{* * * *}$ & $-85^{* * * *}$ \\
\hline DA10D & & & & & & & & \\
Irrigué & 1,04 & 1,13 & 1,07 & 0,82 & 1,14 & 1,00 & 1,12 & 0,98 \\
Stressé & 1,08 & 0,84 & 1,19 & 0,94 & 1,08 & 0,83 & 0,41 & 0,08 \\
\hline Ecart $(\%)$ & +4 & -26 & +11 & +15 & -5 & -18 & $-64^{* * *}$ & $-92^{* * * *}$ \\
\hline${ }^{*}=$ seuil $\alpha<0,01 ;{ }^{* * * *}=$ seuil $\left.\alpha<0,0001\right)$ & & & & &
\end{tabular}

\section{DISCUSSION}

\section{SATISFACTION DES BESOINS EN EAU}

L'installation du stress hydrique a été rapide et bien marquée au cours de la phase de dessèchement. Elle a entraîné une baisse sévère du taux de satisfaction des besoins en eau des jeunes plants, qui a atteint des valeurs particulièrement faibles $(T T R<5 \%)$ à la fin de la troisième semaine. En définitive, la transpiration relative est apparue comme un indicateur efficace de l'intensité de la sécheresse dans nos conditions expérimentales. Cette précision revêt une importance particulière, car au champ, la transpiration relative n'atteint presque jamais des valeurs aussi faibles. En Côte d'Ivoire, Dufrène et al. (1993) ont enregistré au champ sur les sables tertiaires ferralitiques fortement désaturés, des transpirations relatives comprises entre 81 et $56 \%$ au cours de 3 saisons sèches successives modérées. Dans des conditions plus drastiques, elle a atteint $20 \%$ selon les mêmes auteurs. Wormer et Ochs (1959) ont mesuré des taux de satisfaction des besoins en eau inférieurs à $10 \%$ sur des palmiers soumis au dessèchement au Bénin. Ces auteurs ayant travaillé dans des conditions proches des nôtres (pots de culture), on peut penser qu'en dessous de $10 \%$, le rapport ETR/ETM traduit un état de déshydratation avancée des plantules, bien plus qu'une tension normale de sécheresse. Cela s'est ressenti sur le potentiel hydrique qui a fortement baissé pour atteindre des valeurs très négatives, souvent proches de - $4 \mathrm{MPa}$. Dans certains cas, on a même observé des valeurs plus faibles et correspondant à une gamme de pression dépassant les limites de lecture de l'appareil utilisé. Villalobos et al. (1992) ont trouvé des valeurs comprises entre $-1,5$ et $-1,95$ MPa pendant la saison sèche sur des plantules de 10 mois. De même, Harun (1997) a obtenu des valeurs proches de - 2,1 MPa sur des palmiers de 1 an, après 8 semaines de stress. 
Visuellement, les plantules avaient atteint un état de flétrissement irréversible. L'absence de différence significative entre les potentiels hydriques observée entre les deux traitements hydriques, après seulement $4 \mathrm{j}$ de réhydratation traduit une capacité de récupération des plantules pour ce paramètre.

\section{EFFETS DU DEFICIT HYDRIQUE SUR LES ECHANGES GAZEUX}

La photosynthèse mesurée sur des plantules irriguées âgées de 4 mois a atteint des valeurs proches de $15 \mu \mathrm{mol} \mathrm{m} \mathrm{m}^{-2} \mathrm{~s}^{-1}$. Ces valeurs représentent près du double de celles obtenues récemment par Jeyakumar et Kallarackal (1999) sur des plantules de 8 mois $\left(7,62 \mu \mathrm{mol} \mathrm{m}^{-2} \mathrm{~s}^{-1}\right)$. Cette grande différence est à mettre en relation probablement avec les conditions expérimentales. En effet, l'expérimentation s'est déroulée sous serre, avec des conditions proches de l'optimum pour le palmier à huile comme définies par Dufrène (1989) : PAR = $1200 \mu \mathrm{mol} \mathrm{m} \mathrm{m}^{-2} \mathrm{~s}^{-1}$; température foliaire $=$ $30^{\circ} \mathrm{C},\left[\mathrm{CO}_{2}\right]=350 \mathrm{vpm}$, humidité relative $>=$ $65 \%$ et VPD $<1,8 \mathrm{KPa}$. La similitude de ces résultats avec ceux de Dufrène (1989 et 1993) sur des palmiers de 6 mois (entre 16 et $19 \mu \mathrm{mol} \mathrm{m}{ }^{-2} \mathrm{~s}^{-1}$ ) peut être considérée comme la réponse maximale de la photosynthèse des jeunes plants de palmier à huile placées dans les conditions optimales. Ceci confirme ainsi la place de cette plante comme l'une des plus efficientes en C3 (Lamade et al., 1996).

Le manque d'eau a été un facteur essentiel de la limitation de l'assimilation chlorophyllienne. La tendance à "l'évitement" du palmier à huile vis à vis de la sécheresse observée antérieurement par Maillard et al. (1974), Adjahoussou (1983), Carvalho (1991) et Cornaire et al. (1994), s'est encore vérifiée. Grâce à l'efficacité de son système stomatique, la plante ajuste précocement son potentiel photosynthétique. Cependant, d'autres facteurs non stomatiques semblent jouer un rôle important surtout dans les premiers instants de d'apparition du stress hydrique. En effet jusqu'au $4^{\mathrm{e}} \mathrm{j}$ de stress, la baisse de la photosynthèse n'a été que de $12 \%$, alors que la conductance a été réduite de $22 \%$. Cela pourrait être due à une résistance stomatique inférieure à la somme des résistances de la couche limite, du mésophile et de carboxylation pendant cette période, comme en témoignent des valeurs de concentration interne en $\mathrm{CO}_{2}$ élevées (données non présentées). L'effet limitant de l'ouverture des stomates ne s'est manifesté que pour des conductances inférieures à 0,9 mole. Ceci expliquerait en partie, la difficulté évoquée par certains auteurs pour sélectionner des variétés à partir de ces deux critères physiologiques (photosynthèse et conductance stomatique), malgré une bonne corrélation. Des études plus approfondies sur la contribution des causes non stomatiques à la limitation de l'assimilation chlorophyllienne pourraient permettre de répondre plus précisément à cette question.

Le génotype LM404D (sensible) a été le plus affectée par le stress hydrique. Cela provient sans doute d'une mauvaise gestion de l'eau et d'une faible capacité à ajuster ses pertes lorsque les conditions deviennent de plus en plus drastiques. En effet, les plantules issues de ce matériel ont présenté des valeurs de transpiration régulièrement plus élevées (données non présentées) par rapport à DA10D (tolérant). Ce résultat a, par ailleurs, été confirmé par la relation photosynthèse-conductance. Le comportement distinctif des deux génotypes confirme le constat fait par Cornaire et al. (1994) selon lequel, en situation de stress, les croisements sensibles gardent plus longtemps leurs stomates ouverts.

Après 4 j de réhydratation, la récupération des plantules n'a pas été complète. Elle n'a été que de $18 \%$ pour la photosynthèse. Une telle étude trouve son intérêt au champ où, les jeunes palmiers sont souvent confrontés à des cycles successifs de sécheresse. Au plan morphologique, la majorité des plants avaient retrouvé un aspect "normal" après les $4 \mathrm{j}$ de réhydratation. Cet état s'est amélioré au fil du temps.

\section{ASSIMILATION DE $\mathrm{CO}_{2}$ ET INTEGRITE DES CHLOROPLASTES}

L'activité photosynthétique a été sérieusement réduite par la sécheresse. En plus des facteurs déjà évoqués, cette limitation peut être due à une altération des centres réactionnels de l'appareil chloroplastique. En effet, Jefferies (1992) a observé une différence ${ } P 0$ chez 7 variétés de patate soumises au stress hydrique. Pour Havaux (1992), la baisse de $\varphi E 0$ s'expliquerait par le fait qu'en conditions de stress hydrique, l'inhibition de l'activité photosynthétique réduit fortement la demande en ATP et NADPH. Ceci conduit à un ralentissement du flux linéaire d'électrons dans les membranes des thylakoïdes. La plus grande sensibilité de LM404D semble résider au niveau du transfert 
des électrons ( $\Psi$ 0). II pourrait s'agir là d'une altération réversible des plastoquinones car la réhydratation des plantules a permis d'améliorer ce transfert. Dans nos conditions expérimentales, le rendement quantique maximal $(\varphi \mathrm{P} 0)$, le rendement du transfert des électrons $(\Psi 0)$ et l'indice de vitalité des chloroplastes (SFI), ont permis de distinguer précocement les deux génotypes, tout juste une semaine après l'installation du stress hydrique. Sous réserve de validation sur une gamme de génotypes plus large, ces trois paramètres peuvent être proposés comme critères de sélection du palmier à huile en réponse à la sécheresse au jeune âge. Enfin, un couplage de cette activité photochimique globale avec les autres facteurs de limitation de la photosynthèse reste à préciser.

\section{CONCLUSION}

Malgré une assez bonne corrélation, les paramètres d'échanges gazeux mesurés (photosynthèse et conductance stomatique) n'ont pas permis de distinguer le comportement des deux génotypes de palmier à huile vis-à-vis de la sécheresse. Par contre, la fluorescence chlorophyllienne s'est révélée comme un paramètre efficace de discrimination du palmier à huile au jeune âge. En effet elle a permis de distinguer les deux génotypes après seulement $7 \mathrm{j}$ de stress. Ce résultat présente un intérêt certain qui pourrait servir d'outil de travail aux sélectionneurs pour la mise en place d'un test précoce de sélection du palmier à huile tolérant à la sécheresse. Sa précision, sa fiabilité et sa facilité de mise en œuvre sont des atouts en terme de gain de temps et d'espace. L'ensemble de ces résultats constitue toutefois une étape préliminaire devant servir au criblage des génotypes de palmier à huile tolérants à la sécheresse dès le jeune âge. Des investigations complémentaires portant sur des conditions proches du champ devront permettre de confirmer ces recherches et de les étendre à l'identification de marqueurs de stress chez le palmier à huile.

\section{REMERCIEMENTS}

Les auteurs remercient l'agence de coopération allemande (Deutscher Akademischer Austauschdienst) pour le soutien financier qui a permis de réaliser cette étude.

\section{REFERENCES}

Adam J. 1910. Le palmier à huile. Challamel, Paris, $243 \mathrm{p}$.

Adjahoussou D. F. 1977. Contribution à l'étude des caractères physiologiques de résistance à la sécheresse du palmier à huile (Elaeis guineensis Jacq.). Thèse de Doctorat de $3^{\mathrm{e}}$ cycle, Université Paris VII, Paris, $145 \mathrm{p}$.

Adjahoussou D. F. 1983. Contribution à l'étude de la résistance à la sécheresse chez le palmier à huile (Elaeis guineensis Jacq.) Thèse de Doctorat d'Etat. Université Paris VII, Paris, 203 p.

Caliman J. P. 1992. Oil palm and water deficit, production, adapted cropping techniques. Oléagineux 47 (5) : 205 - 216.

Carvalho R. de C. 1991. Mécanismes de résistance à la sécheresse chez les plantes jeunes et adultes de palmier à huile. Thèse de Doctorat, Université de Paris sud, Paris, (nbre de pages.)

Cerovic Z. G., Goulas Y., Gorbunov M., Briantais J M., Camenen M. et I. Moya. 1996. Fluorosensing of water stress in plants : diurnal changes of the mean lifetime and yield of chlorophyll fluorescence, measured simultaneously anda $t$ distance with $\tau$-LID$\mathrm{AR}$ and modified PAM fluorometer in maize, sugar beet and kalanchoe. Remote Sens Environ. 58 : 311 - 321.

Corley R. H. V. 1983. Photosynthesis and age of oil palm leaves. Photosynthetica 17 (1) : $97-100$.

Cornaire B., Daniel C., Zuily-Fodil Y. et E. Lamade. 1994. Le comportement du palmier à huile sous stress hydrique. Données du problème, premiers résultats et voie de recherches. Oléagineux $49: 1-12$. 
Cruizat P. 1974. Détermination des pertes en eau subies par les différents organes d'une plante soumise au dessèchement. Ann. Agron., 4 : 539 - 554.

Devuyst A. 1948. Influence des pluies sur les rendements du palmier à huile enregistrées à la station de La Mé de 1938 à 1946. Oléagineux 3 (3) : 137 - 144.

Diouf M. 1993. L'eau dans le système sol - plante - athmosphère : Concepts, méthodes d'étude. Application à Acacia tortilis (Forsk.) Hayne subsp. Raddiana (Savi) Brenan var. raddiana Brenan, (Mimosaceae). Mémoire de DEA, Université Cheick Anta Diop, DAKAR, $131 \mathrm{p}$.

Dufour O., Frère J. L., Caliman J. P. et P. Hormus. 1988. Présentation d'une méthode simplifiée de prévision de la production d'une plantation de palmier à huile à partir de la climatologie. Oléagineux, 43 : 272 - 278.

Dufrène E. 1989. Photosynthèse, consommation en eau et modélisation de la production chez le palmier à huile. Thèse de Doctorat. Université de Paris-Sud, Orsay, 155 p.

Dufrène E. and B. Saugier. 1993. Gas exchange of oil palm in relation to light, vapour pressure deficit, temperature and leaf age. Functional Ecology 7 : 97 - 104.

Dufrène E., Dubos B., Rey H., Quencez P. and B. Saugier. 1992. Changes in evapotranspiration from an oil palm stand (Elaeis guineensis Jacq.) exposed to seasonal soil water deficits. Acta Oecologica 13 (3) : 299 - 314.

Flexas J., Briantais J. M., Cerovic Z., Medrano H. and I. Moya. 2000. Steady-state and maximum chlorophyll fluorescence responses to water stress in grapevine leaves : a new remote sensing system. Remote Sens Environ 73 : 283 - 297.

Harun M. H. 1997. Proline accumulation in the leaves of water stressed oil palm (Elaeis guineensis Jacq.) seedlings. Elaeis 9 (2) : 93 - 99.

Havaux M. 1992. La fluorescence de la chlorophylle in vivo : quelques concepts appliqués à l'étude de la résistance de la photosynthèse aux contraintes de l'environnement. In (auteurs - initiales (Eds.).) Tolérance à la sécheresse des céréales en zone méditerranéenne. Diversité génétique et amélioration variétale. Montpellier (FRANCE). Ed. INRA, Paris : pp 19 - 29.

He D. and G. E. Edwards. 1996. Evaluation of the potential to measure rates in $\mathrm{C}_{3}$ plants
(Flaveria pringlei and Oryza sativa) by combining chlorophyll fluorescence analysis and a stomatal conductance model. Plant Cell Environ 19 : 1272 - 1280.

Jacquemard J. C. 1995. Le palmier à huile. Le Technicien d'Agriculture Tropicale. Maisonneuve et Larose (33), 207 p.

Jefferies R. A. 1992. Effects of drought on chlorophyll fluorescence in potato (Solanum tuberosum L.). I. Plant water status and the kinetics of chlorophyll fluorescence. Potato research $35: 25-34$.

Jeyakumar P. and J. Kallarackal. 1999. Physiological effects of water stress and certain ameliorants in tenera oil palm seedlings. Journal of Plantation Crop 27 (3) : 212 - 215.

Konan J. L. 1993. Résistance variétale à la sécheresse. Etude du stress hydrique sur plants de pépinière et cocotiers adultes (Cocos nucifera L). DEA, Université de Cocody, Abidjan, Côte d'Ivoire, 56 p.

Krüger G. H. J., Tsimilli-Michael M. and R. J. Strasser. 1997. Light stress provokes plastic and elastic modifications in structure and function of photosystem II in camelia leaves. Physiol. Plant. 101 : 265 - 277.

Lamade E. 1993. Echanges gazeux et bilan carboné chez le palmier à huile au sud-Benin. 1. Echanges gazeux foliaires. Document interne, CIRAD, Montpellier, $40 \mathrm{p}$.

Lamada E., Bonnot F. and Z. Poeloengan. 1996 : Modelling photosynthesis and stomatal response of oil palm clones to environmental conditions in north Sumatra (Indonesia). Implication for plant breeding. Proceedings of the 1996 PORIM International Palm Oil Congress (Agriculture) : 87 - 96.

Maillard G., Daniel C. et R. Ochs. 1974. Analyse des effets de la sécheresse sur le palmier à huile. Oléagineux $29: 8-9$.

NGuettia R. Y., Dofissi S. O., Ballo K. et L. Fondio. 1995. Déclin de la pluviosité en Côte d'Ivoire : Impact éventuel sur la production du palmier à huile. Sécheresse 6 (3) : $265-271$.

Nouy B., Baudoin L.., Djegui N. et A. Omore. 1999. Le palmier à huile en conditions hydriques limitantes. Plantations Recherche Développement : $31-40$.

Quencez P. 1996. La culture du palmier à huile en Afrique intertropicale : les conditions du milieu physique. OCL 3 (2) : 116 - 118.

Repellin A., Laffray D., Daniel C., Braconnier S. and Y. Zuily-Fodil. 1997. Water relations and gas 
exchange in young coconut palm (Cocos nucifera L.) as influenced by water deficit. Canadian Journal of Botany $17: 18$ - 27.

SAS Institut Inc., 1989. SAS/STAT® User guide, Version 6, fourth edition (1), Cary, 943 p.

Strasser B. J. et R. J. Strasser. 1995. Measuring fast fluorescence transients to address environmental questions : the JIP test pp 977-980, In : (P. Mathis ed.), Photosynthesis : from light to biosphere, Kluwer Academic Publishers, Dordrecht, vol. 5 : 977 - 980.
Surre C. 1968. Les besoins en eau du palmier à huile. Calcul du bilan de l'eau et des applications pratiques. Oléagineux 23 (3) : 165 - 167.

Villalobos E., Umana C. H. et C. Chinchilla. 1992. Etat d'hydratation du palmier à huile en réponse à la sécheresse au Costa Rica. Oléagineux 47 (5) : 217 - 223.

Wormer Th. M. et R. Ochs. 1959. Humidité du sol, ouverture des stomates et transpiration du palmier à huile et de l'arachide. Oléagineux 14 (10) : 571 - 580. 